\title{
Prevalencia de problemas emocionales y conductuales en una muestra de adolescentes mexicanos
}

\author{
Julio Cesar Hernández Navor \\ Rosalinda Guadarrama Guadarrama \\ Marcela Veytia López \\ Octavio Márquez Mendoza \\ Universidad Autónoma del Estado de México, Toluca, México
}

\begin{abstract}
Resumen
Los trastornos mentales son considerados como un problema de salud pública, debido a su alta prevalencia. Por ello, el objetivo de la presente investigación fue obtener la Razón de Prevalencia (RP) de los problemas emocionales y conductuales de acuerdo al sexo en 653 adolescentes mexicanos con un rango de edad de 15 a 18 años, a través de la Escala de Problemas Emocionales y Conductuales validada por Andrade, Betancourt y Vallejo (2010). Los resultados obtenidos reportan que las mujeres tienen el 54\% más riesgo de presentar problemas internalizados y externalizados en comparación con los hombres. Finalmente se enfatiza la necesidad de considerar las diferencias de cada sexo al evaluar los problemas emocionales y conductuales, así como en el desarrollo de estrategias encaminadas a la promoción de su salud.
\end{abstract}

Palabras clave: Adolescencia; Problemas emocionales; Problemas conductuales; YSR; Psicopatología.

\section{Prevalência de problemas emocional e comportamento em uma amostra de adolescentes mexicanos}

\section{Resumo}

Os transtornos mentais são considerados como um problema de saúde pública devido à sua alta prevalência. O objetivo deste trabalho foi fazer uma comparação por sexo da presença de problemas emocionais e comportamentais em 653 adolescentes mexicanos com uma faixa etária de 15 a 18 anos, através da Escala de Problemas Emocionais e Comportamentais, validada por Andrade, Betancourt e Vallejo (2010). Os resultados revelaram que as mulheres são 54\% mais risco de internalização e externalização de problemas em relação aos homens. Finalmente, é enfatizada a necessidade de considerar as diferenças de cada sexo na avaliação de problemas emocionais e comportamentais, bem como no desenvolvimento de estratégias voltadas para a promoção de sua saúde.

Palavras-chave: Adolescência; Problemas emocionais; Problemas de comportamento; YSR; Psicopatologia.

\section{Prevalence of emotional and behavioral problems in a sample mexican adolescents}

\begin{abstract}
Today, mental disorders are considered as a public health problem due to its high prevalence. Therefore the aim of this research was to make a comparison by gender of the presence of emotional and behavioral problems in 653 Mexican adolescents with an age range of 15 to 18 years, through the Problem Scale Emotions and Behavioral validated by Andrade, Betancourt and Vallejo (2010). The results show that women have a 54\% higher risk of internalized and outsourced problems compared to men. Finally, the need to consider the differences of each sex in evaluating emotional and behavioral problems, as well as in the development of strategies aimed at promoting their health, is emphasized.
\end{abstract}

Keywords: Adolescence; Emotional problems; Behavioral problems; YSR; Psychopathology. 


\section{Introducción}

De acuerdo con la Organización Panamericana de la Salud y la Organización Mundial de la Salud (OMS, 2011) los trastornos mentales corresponden al $12 \%$ del total de las enfermedades a nivel mundial. Afectando especialmente a la adolescencia y adultez temprana (Campo \& Cassiani, 2008).

En el caso de la adolescencia, los distintos cambios biopsicosociales, hacen que esta población esté más proclive a padecer problemas emocionales y conductuales al estar en una etapa de transición de la infancia a la edad adulta (Lozano, 2014; Nieto, 2013; Santillano, 2009). Provocando que se incremente el interés por el estudio de su salud mental en las últimas décadas (Coleman, 2008).

En este sentido, uno de los primeros sistemas en desarrollar una taxonomía de la psicopatología específicamente para niños y adolescentes, diferente a las nosologías planteadas para los adultos, ha sido el Sistema de Evaluación Basado Empíricamente de Achenbach (ASEBA), el cual tiene como propósito evaluar de una forma integral comportamientos adaptativos y desadaptativos, convirtiéndolo en uno de los más utilizados para investigación clínica y diagnóstico a nivel mundial (Achenbach et al., 2008; Andrade et al., 2010; Bordin et al., 2013).

Entre los instrumentos que conforman dicho sistema se encuentra el Youth Self Report, el cual evalúa los problemas reportados por los propios adolescentes, $\mathrm{y}$ ha sido utilizado con diferente fin en variadas investigaciones por ejemplo: Alarcón y Bárrig (2015) reportaron que los síndromes con la media más alta para los adolescentes peruanos son: Conducta Agresiva y Ansiedad/depresión.

Por su parte, Fernández y Fernández (2012), encontraron una diferencia estadística significativa en todas las conductas problema en una muestra de adolescentes venezolanos (institucionalizados y grupo control), siendo los adolescentes institucionalizados los que tienen las puntuaciones más altas. Por otra parte, Navarro y García (2012), encontraron que existe una fuerte relación con la sintomatología externalizante y el rendimiento académico. De esta manera, López y Fleixinós (2001) investigaron la relación entre el consumo de alcohol y la psicopatología asociada a dicho consumo.

Con relación a la variable sexo, Zubeidat, Fernández, Ortega, Vallejo y Sierra (2009) encontraron una diferencia estadística significativa en todas las conductas problema, es decir, los hombres tienen la media más alta a comparación de las mujeres. Mientras Alarcón y Bárrig (2015) reportan que existe una diferencia estadística significativa en los problemas emocionales, siendo las mujeres las que puntúan más alto. Ambas puntuaciones coinciden con los resultados encontrados por Zanini y Forns (2004), sin embargo, estos dos últimos autores no reportan una diferencia estadística significativa.

En México, la investigación utilizando este mismo instrumento ha sido escasa, hasta el momento solo se tiene la traducción y adaptación echa por Valencia y Andrade (2005), en una población de 9 a 15 años. Posteriormente Vallejo et al. (2008), determinaron los principales problemas que presentaba una muestra de adolescentes de bachillerato con la versión ajustada del YSR, señalando una diferencia estadística significativa entre hombre y mujeres en los factores de Depresión, Problemas Internalizados y Pensamiento, siendo las mujeres las que puntúan por arriba de los hombres.

Más adelante Andrade et al., (2010) determinaron la estructura factorial y confiabilidad del mismo instrumento ampliando la edad de aplicación (12 a 18 años), en dicha investigación se encontró una diferencia estadística significativa en los síndromes de depresión, conducta agresiva, problemas somáticos y lesiones auto-infligidas.

Con el fin de seguir con esta línea de investigación, el objetivo del presente estudio es obtener la RP de los Problemas Emocionales y Conductuales en adolescentes mexicanos de acuerdo al sexo, a través de la escala validada por Andrade et al., (2010). Si bien existe evidencia científica de puntuaciones promedio, no hay antecedentes que calculen RP en población mexicana.

\section{Método}

La presente investigación cuantitativa es de tipo transversal analítico, ya que pretende obtener la Razón de Prevalencia de los Problemas Emocionales y Conductuales por sexo en una muestra de adolescentes mexicanos.

\section{Participantes}

Se utilizó una muestra no probabilística intencional con un total de 653 estudiantes de preparatoria (Nivel 4 de acuerdo con la CINE-2011 de la UNESCO) del Valle de Toluca, Estado de México. Del total de los participantes $279(42.7 \%)$ son hombres y 374 (57.3\%) son mujeres, ambos sexos presentaron una media de edad de 15.3216 con un D.E $=.5489$.

Los criterios de inclusión fueron que al momento de la investigación los alumnos participantes cursaran el segundo semestre de preparatoria con un rango de edad de entre 15 a 16 años, y por último que presentaran en forma física el formato original de asentimiento infor- 
mado y consentimiento informado debidamente firmado por ellos mismo y sus padres de familia o tutores.

\section{Instrumento}

Para la presente investigación se utilizó la Escala de Problemas Emocionales y Conductuales de Andrade et al., (2010). Dicha escala consta de 38 reactivos y siete síndromes: 1 ) Depresión ( 8 reactivos, $\mathrm{a}=0.89$ ), 2) Rompimiento de Reglas (7 reactivos, $\mathrm{a}=0.83$ ), 3) Consumo de Alcohol y Tabaco (4 reactivos, $\mathrm{a}=0.76)$, 4) Problemas Somáticos (5 reactivos, $\mathrm{a}=0.77), 5$ ) Conducta Agresiva (8 reactivos, $\mathrm{a}=0.82$ ), 6) Problemas de Pensamiento ( 3 reactivos, $a=0.72$ ) y 7) Lesiones Auto-inflingidas (3 reactivos, $a=0.70$ ). También cuenta con dos síndromes de banda ancha que evalúan problemas Internalizados y Externalizados que se componen de la siguiente manera:

- Problemas Internalizados: compuesta por los factores de Depresión, Problemas Somáticos, Lesiones Auto-infligidas y Problemas de Pensamiento.

- Problemas Externalizados: compuesto por los factores de Rompimiento de Reglas, Conducta Agresiva y Consumo de Alcohol y Tabaco.

Los puntos de corte para el instrumento son: puntuaciones menores de 50 puntos es una presencia de problemáticas baja, puntuaciones entre 50 a 70 puntos la presencia de conductas problema es moderada y por último las puntuaciones mayores a 70 puntos la presencia de conductas problema es alta.

\section{Procedimiento}

Para la aplicación del instrumento de investigación se realizaron sesiones informativas con los participantes, en ellas se les mencionó los objetivos, alcances y beneficios de la investigación. De igual forma se les mencionó que la participación era de forma voluntaria, que no existían riesgos físicos y psicológicos al participar y la confidencialidad de sus datos.

Lo anterior quedó expresado en un asentimiento informado y consentimiento informado firmado por sus padres y por ellos de acuerdo a la Declaración de Helsinki al ser menores de edad. Al obtener el asentimiento informado y el consentimiento informado firmado por los padres de familia o tutor y el propio alumno se continuó con la aplicación del instrumento de manera individual y grupal dentro de las instalaciones de las diferentes preparatorias de la UAEMéx, con una duración de 25 minutos aproximadamente.

\section{Análisis Estadístico}

Para determinar la RP se empleó la regresión logística binaria, siendo la variable dependiente los problemas emocionales y conductuales y la variable independiente el sexo, a un nivel de significancia $\mathrm{p}<.05$.

\section{Resultados}

Como se puede observar en la Tabla 1, existe diferencia estadística significativa entre hombres y mujeres en los factores de Depresión, Problemas Somáticos, Problemas de Pensamiento y lesiones Auto-infligidas. Dado que los intervalos de confianza no contienen al valor 1 , y la $\mathrm{p}<.05$.

Con respecto al factor de Depresión, las mujeres tienen $31 \%$ más riesgo (Odds Ratio [OR] $=.317$,IC95\%, 208-.482) de presentar síntomas como: sentir que nadie las quiere, sentirse inferior o creer no valer nada, sentirse solas/triste/deprimidas/infelices, no comprendidas, culpables, confundidas y llorar mucho en comparación con los hombres.

Esto mismo se puede observar en el factor de Problemas Somáticos en donde las mujeres tienen el 42\% más riesgo (Odds Ratio [OR] $=.423$,IC95\%, .288-.621) de presentar los siguientes problemas: nauseas/vómito, dolor de cabeza, dolor de estómago y mareo en comparación con los hombres.

Con respecto al factor de Problemas de Pensamiento las mujeres tienen $64 \%$ más riesgo (Odds Ratio $[\mathrm{OR}]=.649$,IC95\%,.432-.975) en comparación con los hombres de presentar comportamientos o ideas que otras personas piensan que son raras y repetirlas una y otra vez. Finalmente en el factor de Lesiones Auto-infligidas se observa que las mujeres tienen el 28\% más de riesgo (Odds Ratio $[\mathrm{OR}]=.284, \mathrm{IC} 95 \%, .165-.489)$ de pensar e intentar suicidarse en comparación con los hombres (ver Tabla 1).

Como se puede observar en la Tabla 2, con respecto al valor total de la escala y los síndromes de banda ancha, existe diferencia estadísticamente significativa solamente en el total de la prueba, así como, en los Problemas Internalizados, siendo mayor en las mujeres con respecto a los hombres.

Es decir, las mujeres tienen $41 \%$ más riesgo (Odds Ratio $[\mathrm{OR}]=.416$, IC95\%,.282-.615) de presentar en mayor medida estrés interno o comportamientos dirigidos al interior que provocan inadaptabilidad y daños a uno mismo a comparación de los adolescentes hombres.

Por otra parte, para el total de la escala las mujeres tienen el 54\% más probabilidad (Odds Ratio $[\mathrm{OR}]=.544$, IC95\%, .381-.777) de presentar problemas emocionales y conductuales en comparación con los hombres (ver Tabla 2). 
TABLA 1

RP a partir del análisis de regresión logística binaria para cada uno de los problemas emocionales y conductuales de acuerdo al sexo

\begin{tabular}{|c|c|c|c|c|c|c|c|c|}
\hline \multirow[t]{2}{*}{ Factor } & \multicolumn{2}{|c|}{$\begin{array}{c}\text { Hombres } \\
n=279\end{array}$} & \multicolumn{2}{|c|}{$\begin{array}{l}\text { Mujeres } \\
n=374\end{array}$} & \multirow{2}{*}{$\begin{array}{c}P R \\
\text { log-binomial }\end{array}$} & \multirow[t]{2}{*}{ Sig. } & \multirow[t]{2}{*}{ OR } & \multirow[t]{2}{*}{$95 \% I C$} \\
\hline & Media & D.E & Media & D.E & & & & \\
\hline Depresión & 1.47 & 0.47 & 1.83 & 0.68 & -1.150 & .001 & .317 & $(.208-.482)$ \\
\hline Rompimiento de Reglas & 1.88 & 0.54 & 1.88 & 0.55 & -.036 & .845 & .675 & $(.675-1.380)$ \\
\hline Consumo de Alcohol y Tabaco & 1.42 & 0.54 & 1.37 & 0.51 & .243 & .194 & 1.275 & $(.884-1.839)$ \\
\hline Problemas Somáticos & 1.61 & 0.56 & 1.87 & 0.60 & -.860 & .001 & .423 & $(.288-.621)$ \\
\hline Problemas de Pensamiento & 1.76 & 0.70 & 1.90 & 0.75 & -.433 & .037 & .649 & $(.432-.975)$ \\
\hline Lesiones Auto-infligidas & 1.12 & 0.33 & 1.30 & 0.56 & -1.257 & .001 & .284 & $(.165-.489)$ \\
\hline Conductas Agresivas & 1.70 & 0.54 & 1.66 & 0.53 & .226 & .205 & 1.254 & $(.884-1.779)$ \\
\hline
\end{tabular}

TABLA 2

RP a partir del análisis de regresión logística binaria para síndromes de banda ancha de acuerdo al sexo

\begin{tabular}{lcccccccc}
\hline & \multicolumn{2}{c}{$\begin{array}{c}\text { Hombres } \\
n=279\end{array}$} & \multicolumn{2}{c}{$\begin{array}{c}\text { Mujeres } \\
n=374\end{array}$} & PR & Sig. & OR & 95\% IC \\
& Media & $D E$ & Media & DE & log-Binomial & & \\
Internalizados & 7.14 & 1.90 & $\mathbf{8 . 4 2}$ & 2.52 & -.876 & .001 & .416 & $(.282-.615)$ \\
Externalizados & 10.84 & 2.86 & 10.66 & 2.85 & .252 & .152 & 1.287 & $(.911-1.817)$ \\
Total & 61.12 & 14.17 & $\mathbf{6 5 . 7 0}$ & 16.75 & -.608 & .001 & .544 & $(.381-.777)$ \\
\hline
\end{tabular}

\section{Discusión}

El objetivo de la presente investigación fue obtener la Razón de prevalencia de los problemas emocionales y conductuales en una muestra de adolescentes mexicanos de acuerdo al sexo, los resultados mostraron que si existen diferencias estadísticas significativas en los factores de Depresión, Problemas somáticos, Problemas de pensamiento y Lesiones auto-infligidas, siendo las mujeres las que tienen $41 \%$ más riesgo de presentar problemas internalizados en comparación con los hombres.

Dichos resultados son en parte iguales a los reportados en investigaciones realizadas por Vallejo et al., (2008) y Andrade et al., (2010) en población mexicana, es decir, de los 5 síndromes que mencionan las autoras en sus investigaciones, en esta investigación el factor de Conducta Agresiva no presentó diferencia estadística significativa. Resultado que coincide con los reportados por Hernández (2016) en adolescentes del estado de México.

No obstante, es importante señalar que los autores anteriores no obtuvieron la Razón de prevalencia de cada factor y síndrome de banda ancha, ya que sus resultados reportaron promedios de cada uno de los factores, sin embargo, nos permite vislumbrar que la presencia de problemas emocionales se sigue presentando en mayor razón en las mujeres. Es decir tienen mayor probabilidad de presentar reacciones emocionales y cognitivas que van dirigidas hacia el propio sujeto de manera negativa (Achenbach y Edelbrock, 1987).

Una posible explicación a los resultados encontrados podría ser lo señalado por Ramos (2014), al mencionar que la salud mental de las mujeres es más vulnerable a ser afectada por distintos factores sociales entre los que se destacan la desigualdad, el aislamiento social, bajo nivel socioeconómico, violencia, entre otros. Aunado a esto la influencia de los estereotipos de cada género en las vivencias emocionales, en la cual se le adjudica a las mujeres ser más sensibles que los hombres (Amurrio, Larrinaga, Usategui y Del Valle, 2012; Etxebarria, 2003).

Por otra parte, si bien no se encontraron diferencias estadísticas significativas en los factores que conforman el síndrome de banda ancha de Problemas Externalizados (Conducta agresiva, Rompimiento de reglas, Consumo de alcohol y tabaco) la RP de dichos factores son mayores en los hombres que en las mujeres, resultados que concuerdan en parte con 
investigaciones ya realizadas en México y a nivel internacional (Alarcón y Bárrig, 2015; Andrade et al., 2010; Hernández, 2016; Vallejo et al., 2008; Zanini y Forns, 2004; Zubeidat et al., 2009).

Lo cual nos permite constatar lo que se señala en la literatura científica, que el estereotipo de los varones va ligado a ser valientes, arriesgados, no mostrar frustración y debilidad, así como reprimir las reacciones afectivas por otras personas (Amurrio et al., 2012). Sin embargo en ellos, los malestares emocionales pueden estar encubiertos por comportamientos adictivos y de riesgo (Ramos, 2014).

Otra de las contribuciones más importantes del presente estudio fue obtener la razón de prevalencia de problemas emocionales y conductuales de forma general, los resultados obtenidos demuestran que nuevamente las mujeres tienen el $54 \%$ más de probabilidad de presentar dichos problemas en comparación con los hombres. Dato que confirma lo señalado por Hernández (2016), al decir que si bien los problemas Externalizados son más fáciles de identificar, estos a su vez, son el resultado de un conglomerado de problemas internalizados.

Lo anterior, acentúa la necesidad de realizar esfuerzos para atender los diferentes problemas internalizados y externalizados por los que atraviesan los adolescentes, debido a que estos determinaran en cierta forma la manera de relacionarse con los demás, disfrutar las vivencias individuales, así como un buen desarrollo en el ámbito social y académico. Más aún, en esta etapa de la vida que tiene como objetivo una adaptación psicosocial (Coleman y Hendry, 2003; Nieto, 2013), en la cual la presencia de los problemas internalizados y externalizados es más certera, como consecuencia de los distintos cambios biopsicosociales que se tienen (Hernández, 2016).

Es por ello que se debe de poner atención en la intensidad y duración de dichos rasgos emocionales y de conducta en los adolescentes, que son clave para el diagnóstico de una posible patología (Calzada, 2013). Ante esto, se sugiere continuar con esta línea de investigación haciendo énfasis en los distintos factores de riesgo que provocan que los adolescentes presenten malestar emocional y problemas de conducta, teniendo en cuenta las características de cada sexo.

Por otro lado, sería conveniente comenzar con el estudio de la validación de los demás instrumentos que conforman el Sistema de Evaluación Basado Empíricamente de Achenbach (ASEBA) en población mexicana con el objetivo de tener más herramientas para un diagnóstico integral de la psicopatología en la población adolescente.

Finalmente se recomienda profundizar en el estudio de los problemas emocionales y conductuales en esta misma población pero con características diferentes por ejemplo: adolescentes de zona rural, institucionalizados, con alguna enfermedad crónica, así como tomar en cuenta algunas variables sociodemográficas como ingresos, numero de hermanos, afiliación parental, entre otras.

Esto nos permitirá tener un mejor conocimiento de esta población y de esta forma generar estrategias encaminadas a mejor su salud mental a través de programas de promoción, prevención e intervención que permitan una reeducación emocional para mejorar su calidad de vida (Guadarrama, Hernández, Veytia y Márquez, 2014). Eje central de nuestra labor como profesionales de la salud.

\section{Referências}

Achenbach, T. \& Edelbrock, C. (1987). The manual for the Youth Self-Report and Profile. Burlington: University of Vermont.

Achenbach, T., Becker, A., Dopfner, M., Heiervang, E., Roessner, V., Steinhausen, H., \& Rothenberger, A. (2008). Multicultural assessment of child and adolescent psychopathology with ASEBA and SDQ instruments: research finding, applications, and future direction. Journal of Psychology and Psychiatry, 49(3), 251-275. https://doi. org/10.1111/j.1469-7610.2007.01867.x

Alarcón, D. \& Bárrig, P. (2015). Conductas Internalizantes y Externalizantes en adolescentes. LIBERABIT, 21(2), 253-259. Recuperado de http://www.redalyc.org/articulo.oa?id=68643124008

Amurrio, M., Larrinaga, A., Usategui, E., \& Del Valle, A. (2012). Los estereotipos de género en los/las jóvenes y adolescentes. Trabajo presentado en el XVII Congreso de estudios Vascos, País Vasco. Recuperado de http://www. euskomedia.org/PDFAnlt/congresos/17/02270248.pdf

Andrade, P., Betancourt, D., \& Vallejo, A. (2010). Escala para evaluar problemas emocionales y conductuales en adolescentes. Ciencias Sociales y Humanidades, 9(9), 37-44. Recuperado de https://dialnet.unirioja.es/servlet/ articulo? codigo $=3705750$

Bordin, I. A., Rocha, M. M., Paula, C. S., Teixeira, M. C., Achenbach, T. M., Rescorla, L.A., Silvares, E. F. (2013). Child Behavior Checklist (CBCL), Youth Self-Report (YSR) and Teacher's Report Form (TRF): an overview of the 
development of the original and Brazilian version. Cad. Saúde Pública, 29(1), 13-28. Recuperado de http://dx.doi. org/10.1590/S0102-311X2013000100004

Calzada, M. (2013). Trastornos psicopatológicos en la adolescencia y líneas de intervención en la familia. In Mingote, C., \& Requema, M. (Eds.). El malestar de jóvenes (pp. 361-366). Madrid: Ediciones Díaz de Santos.

Campo, A. \& Cassiani, C.A. (2008). Trastornos mentales más frecuentes: prevalencia y algunos factores sociodemográficos asociados. Revista Colombiana de Psiquiatría, 37(4), 598-613. Recuperado de http://www.redalyc. org/pdf/806/80637410.pdf

Coleman, J. (2008). Salud y Bienestar emocional. In J. Coleman, L. Hendry \& M. Kloep (Eds.). Adolescencia y Salud (pp. 52-74). México: Manual Moderno.

Coleman, J. C. \& Hendry, L. B. (2003). Psicología de la Adolescencia. Madrid: Morata.

Etxebarria, I., Apodaca, P., Eceiza, A., Fuentes, M. J., \& Ortiz M. J. (2003). Diferencias de género en emociones y en conducta social en la edad escolar. Infancia y Aprendizaje, 26(2), 147-161. https://doi.org/10.1174/021037003321827759

Fernández, M. P. \& Fernández, A. (2013). Problemas de Comportamiento y Competencias Psicosociales en Niños y Adolescentes Institucionalizados. Universitas Psychologica, 12(3), 707-810.

Guadarrama, R., Hernández J. C., Márquez, O., \& Veytia, M. (2014) Significado psicológico del concepto calidad de vida en adolescentes mexicanos. Pensando Psicología, 10(17), 53-60. Recuperado de http://dx.doi.org/10.16925/ pe.v10i17.784

Hernández, J. C. (2016). Problemas Emocionales y Conductuales en una Muestra de Adolescentes de la Ciudad de Toluca. Tesis de Licenciatura. México: Universidad Autónoma del Estado de México.

López, C. \& Fleixinós, M. A. (2001). Psicopatología y Consumo de Alcohol en Adolescentes. Anales de Psicología, $17(2), 177-188$.

Lozano, A. (2014). Teoría de teorías sobre la adolescencia. Última Década, 40(1), 11-36.

Navarro, L. \& García, D. A. (2013). Sintomatología Externalizante y Rendimiento Académico. International Journal of Developmental and Educational Psychology, 1(1), 657-666.

Nieto, F. J. (2013). Acercamiento a una Adolescencia Mediatizada. Adolescencia y Posmodernidad: Malestares, Vacilaciones y Objetivos. México: Fontamara.

Organización Panamericana de la Salud, Organización mundial de la Salud (2011). Informe de la Evaluación del Sistema de Salud Mental en México Utilizando el Instrumento de Evaluación para Sistemas de Salud Mental de la Organización Mundial de la Salud. Recuperado de http://www.who.int/mental_health/who_aims_country_reports/ who_aims_report_mexico_es.pdf

Ramos, L. (2014). ¿Por qué hablar de género y salud mental? Salud Mental, 37(4), 275-281. https://doi.org/10.17711/ SM.0185-3325.2014.032

Santillano, I. (2009). La adolescencia: añejos debates y contemporáneas realidades. Última década, 1(1), 55-77. Recuperado de http://www.redalyc.org/articulo.oa?id=19511968004

Valencia, M. \& Andrade, P. (2005). Validez del Youth Self Report para problemas de conducta en niños mexicanos. International Journal of Clinical and Health Psychology, 5(3), 499-520.

Vallejo, A., Mazadiego, T., Betancout, D., Vázquez, A., Sagahón, M., Méndez, M., Reyes, C. (2008). Problemas externalizados e internalizados en una muestra de adolescentes de bachilleres veracruzanos. Revista de Educación y Desarrollo, 9(1), 15-22.

Zanini, D. S. \& Forns, M. (2004). Coping y psicopatología: comparación entre la muestra general y sub-clínica, Psiquiatría.com, 8(2). Recuperado de http://www.psiquiatria.com/trastornos infantiles/coping-y-psicopatologiacomparacion-entre-adolescentes-de-la-muestra-general-y-sub-clinica/\#

Zubeidat, I., Fernández, A., Ortega, J., Vallejo, M. A., \& Sierra, J. C. (2009). Características psicosociales y psicopatológicas en una muestra de adolescentes españoles a partir del Youth Self Report/11-18. Anales de Psicología, 25(1), 60-60.

Nota:

La presente investigación fue realizada gracias al financiamiento de la Universidad Autónoma del Estado de México

(Registro interno: 3698/2014/CID).

Autores:

Julio Cesar Hernández Navor - Graduado, Universidad Autónoma del Estado de México.

Rosalinda Guadarrama Guadarrama - Doutora, Universidad Autónoma del Estado de México.

Marcela Veytia López - Doutora, Universidad Autónoma del Estado de México.

Octavio Márquez Mendoza - Doutor, Universidad Autónoma del Estado de México.

Endereço para correspondência:

Julio Cesar Hernández Navor

Instituto Literario \# 100 - Centro

50000 - Toluca de Lerdo, Méx

<cesarfacico@gmail.com>

Recebido em: 04.10.2016

Aceito em: 22.08.2017 